\title{
The Role of Prophylactic Vertebroplasty at UIV+1 and Cement-augmented Fenestrated Pedicle Screw for Prevention of PJK/PJF in Patients With Osteoporosis: A Propensity Score-matched Analysis
}

\section{Qi Wang}

PLAGH: Chinese PLA General Hospital

Chi Wang

PLAGH: Chinese PLA General Hospital

Wenhao Hu

PLAGH: Chinese PLA General Hospital

Fanqi Hu

PLAGH: Chinese PLA General Hospital

Weibo Liu

PLAGH: Chinese PLA General Hospital

Teng Li

PLAGH: Chinese PLA General Hospital

\section{Shiqi Cao}

PLAGH: Chinese PLA General Hospital

Xuesong Zhang ( $\square$ zhangxuesong301pla@163.com )

Department of Orthopaedics, the First Medical Center, Chinese PLA General Hospital, Beijing, 100853, China https://orcid.org/0000-0002-6712-1208

\section{Research article}

Keywords: Adult spinal deformity, PJK, PJF, BMD, prophylactic vertebroplasty, cement augmentation, fenestrated pedicle screw

Posted Date: September 15th, 2020

DOI: https://doi.org/10.21203/rs.3.rs-73483/v1

License: (9) This work is licensed under a Creative Commons Attribution 4.0 International License. Read Full License 


\section{Abstract}

Objective: To elucidate the role of prophylactic vertebroplasty (PV) at UIV+1 and cement-augmented fenestrated pedicle screw (CAFPS) in prevention of PJK and PJF.

Background: Cement augmentation at UIV and UIV+1 was found to prevent PJK and PJF. But most studies are retrospective and have a selection bias due to multifactorial etiology of PJF, making it difficult to identify the efficacy of prophylactic cement augmentation.

Methods: We enrolled 208 surgically treated adult spinal deformity (ASD) patients who were followed for at least 2 years, to elucidate whether prophylactic cement augmentation was truly minimizing the risk of PJK and PJF in a uniform population from one center database. Patients were classified into two groups. Two comparable groups were propensity-matched with one to one nearest neighbor matching. The main outcome variables including PJK, PJF, pedicle screw loosening and cement leakage were compared.

Results $\triangle A$ fter propensity score matching, there were 58 propensity-matched patients in group $A$ and $B$ ( $n=29$ in each group) whose parameters including age, BMI, BMD, number of instrumented vertebrae, SVA, spinal-pelvic parameters and the frequency of UIV and LIV were similar. The incidence of PJK showed no significantly statistical difference between group $A$ and $B(10.3 \%$ vs $13.8 \%, p>0.99)$. Compared to group $A$, group B had a higher proportion of patients developing PJF $(24.1 \%$ vs $0 \%, p=0.01)$. Pedicle screw loosening at UIV and (or) LIV showed no difference ( $24.1 \%$ vs $3.4 \%, p=0.052)$ between two groups. In group A, cement leakage was detected in 5 cases via fluoroscopy, but none of them had neurological deficit or pulmonary cement embolism.

Conclusion: Combined application of CAFPS and PV could reduce the incidence of PJF, but it could not prevent the development of PJK in the surgical management of ASD with low BMD or osteoporosis.

\section{Introduction}

Proximal junctional kyphosis (PJK) and proximal junctional failure (PJF) has exerted great challenge on spine surgeons in the management of spinal deformity. Though there is still no consensus on the definition of PJK, it has been proven that PJK is correlated with up back pain and may progress to PJF ${ }^{1,2}$. PJF may require revision surgery for proximal extension of fusion, which is defined as vertebral fracture of uppermost instrumented vertebrae (UIV) or (and) vertebrae above UIV (UIV + 1), pull-out of instrumentation at UIV or (and) sagittal subluxation ${ }^{3}$. The incidence of PJK/PJF varies greatly due to multiple contributing factors including selection of the UIV and lowest instrumented vertebrae (LIV), spino-pelvic parameters, fusion levels, older age, body mass index (BMI), bone mineral density (BMD) and other elements ${ }^{1,2}$. In order to prevent the PJK and PJF after surgical correction of adult spinal deformity (ASD), some surgeons have resorted to the hook-only constructs, hybrid constructs or ligament augmentation $^{4,5}$. 
Low BMD or osteoporosis has been confirmed as an important risk factor of PJF ${ }^{6}$. In order to reduce the incidence of fracture of UIV/UIV +1 , screw loosening and screw pull-out in osteoporotic spine, cement augmentation at UIV and UIV +1 have already been used ${ }^{7-12}$. However, there still exists controversy about whether cement augmentation can reduce the incidence of the PJK/PJF $5,10,13$. Since most studies concerning cement augmentation for prevention of PJK/PJF have a selection bias due to multifactorial etiology of PJK/PJF and the heterogeneity of ASD population between groups, it is difficult to identify the efficacy of prophylactic cement augmentation at UIV and UIV +1 .

Propensity score matching (PSM) statistical method is commonly used to remove confounding bias when comparing treatment effect in observational cohorts, in which randomization cannot be performed ${ }^{14}$. Therefore, we carried out the present study to elucidate the role of prophylactic vertebroplasty (PV) at UIV +1 and cement-augmented fenestrated pedicle screw (CAFPS) in the prevention of PJK/PJF in a propensity score-matched patient cohort.

\section{Methods}

\section{Subject enrollment}

This was a retrospective analysis of data collected from the Department of Orthopedics in our hospital from January 2010 to January 2018. Study inclusion criteria were as the following. Firstly, the age of patients was above 50 years old at the time of surgery. Secondly, instrumented vertebra was over 5 levels, crossed the thoracolumbar junction and ended proximally in the thoracic spine (T11 or above). Thirdly, patients were diagnosed as adult spinal deformity with at least one of the following radiographic parameters: Cobbs' angle $\geq 20^{\circ}$, sagittal vertical axis (SVA) $\geq 5 \mathrm{~cm}$, or pelvic tilt $\geq 25^{\circ}$. Fourthly, patients underwent posterior spinal fusion with bilateral pedicle screws and rods system (titanium alloy constructs) and were followed up for at least two years.

Exclusion criteria: 1) patients with hook and/or sublaminar wiring usage, 2) long-term use of glucocorticoids or renal dysfunction, 3) neurological disorders affecting balance, 4) the use of antiosteoporotic drugs pre- or post-operatively. According to the use of cement-augmented fenestrated pedicle screw (CAFPS) and prophylactic vertebroplasty (PV), patients were classified into two groups (group A: patients underwent CAFPS and PV at UIV + 1, group B: patients underwent screw rod fixation system without cement augmentation) (Fig. 1).

\section{Surgical Protocol Of Group A}

In group A, the indication for CAFPS and PV was dual-energy X-ray absorptiometry (DEXA) scan T-score < -1 and intraoperative assessment of poor bone quality. T-score for the right femoral neck was used to calculate bone mineral density (BMD) in both groups. 
All CAFPS fixation with cement augmentation and PV at UIV +1 was performed by two senior spine surgeons. After confirmation of all screws position via AP and lateral fluoroscopy, cement augmentation was performed via cement delivery cannulas. In all cases of group A, the same polymethylmethacrylate (PMMA) bone cement was applied in a standard fashion as described by previous report ${ }^{15}$. UIV and LIV of all patients were fixed with CAFPS and other surgical segments were fixed using conventional pedicle screws or CAFPS according to intraoperative assessment of poor bone quality. A total of $2 \mathrm{ml}$ high viscosity bone cement was injected into thoracic vertebra bilaterally $(1 \mathrm{ml}$ bone cement through per fenestrated pedicle screw). A total of $3 \mathrm{ml}$ high viscosity bone cement was injected into lumbar vertebra bilaterally ( $1.5 \mathrm{ml}$ bone cement through per fenestrated pedicle screw). PV at UIV +1 was performed using 3 to $4 \mathrm{ml}$ high viscosity cement after CAFPS fixationd. The progress of bone cement injection was visualized with fluoroscopic guidance (Fig. 2).

\section{Data Collection And Radiographic Assessment}

Preoperative data including age, sex, $\mathrm{BMI}$ and $\mathrm{BMD}$ were recorded. The pelvic incidence (PI), pelvic tilt (PT), lumbar lordosis (LL), PI minus LL, sagittal vertical axis (SVA) and thoracic kyphosis (TK) were measured via preoperative and early postoperative radiograph. Location of UIV and LIV was confirmed on postoperative radiograph. Radiographic measurements were performed using validated software (Surgimap ${ }^{\circledR}$ ). Neurological complication, pulmonary embolism and postoperative PJK/PJF were recorded. At the 24-month follow-up, the spine radiographs were obtained for all patients. Another senior spine surgeon independently evaluated screw loosening, PJK and PJF.

PJK was defined when Cobbs' angle between the lower endplate of UIV and the upper endplate of two super-adjacent vertebra changed greater than $10^{\circ}$ between the immediate post-operative and the 2 years follow-up radiograph ${ }^{2,3}$. PJF, which may require revision surgery for proximal extension of fusion, was defined as vertebral fracture of UIV or (and) UIV +1 , pull-out of instrumentation at UIV, and/or sagittal subluxation ${ }^{3}$. Pedicle screw loosening was defined as the clear zone around the pedicle screw exceeded $1 \mathrm{~mm}$ on anteroposterior and lateral radiographs ${ }^{16}$.

\section{Statistical Analysis}

The propensity score matching procedure was performed using SPSS v24.0 (PS Matching Module). Prior to matching, continuous and categorical variables were compared between two groups using $t$-test, chisquare test or Fisher's Exact Test, respectively. Then, propensity score was estimated based on baseline and imaging parameter covariates by using logistic regression. Patients in group A and group B were then matched by one to one nearest neighbor matching. Following propensity score matching, balance of observed covariates of baseline and preoperative and early postoperative imaging parameters were reexamined. Differences between group $A$ and $B$ were compared by unpaired $t$-test, chi-square test or Fisher's Exact Test. The cut-off point of statistical significance was defined as 0.05 . 


\section{Results}

\section{Patient population}

During this study period, 292 patients who were recruited. 31 cases (group A) underwent CAFPS and PV at UIV + 1 during the period of 2016-2018. 261 cases (group B) underwent screw rod fixation system without cement augmentation during the period of 2010-2016. Though some patients in group B had low T-scores, they did not receive cement augmentation because cement augmentation was not commonly used among surgeon before 2015 in our department. Finally, only 208 patients had complete data and were therefore included in this study. Patients were classified into group A (31 cases) and group B (177 cases) (Fig. 1). The demographic data, preoperative and early postoperative spino-pelvic parameters, UIV, LIV and the frequence of instrumented vertebrae and three-column osteotomy were illustrated in Table 1. Prior to matching, there were statistically differences between two groups with regards of age $(p<0.001)$, pre-LL $(p<0.001), \operatorname{BMD}(p<0.001)$, PT $(p<0.05)$ and LIV $(p<0.001)$ (Table 1$)$. Propensity score was estimated based on baseline and imaging parameter covariates (age, BMD, pre-LL, PT, LIV and SVA). 
Table 1

Demographic and radiographic parameters of pre-matched and propensity score-matched patients

\begin{tabular}{|c|c|c|c|c|c|c|c|}
\hline \multicolumn{2}{|l|}{ Variable } & \multicolumn{2}{|l|}{ All cases } & \multirow{3}{*}{$p_{\text {value }}$} & \multicolumn{2}{|c|}{ Matched group* } & \multirow{3}{*}{$\begin{array}{l}p \\
\text { value }\end{array}$} \\
\hline & & \multirow{2}{*}{$\begin{array}{l}\text { Group A } \\
(\mathrm{n}=31 \\
\text { cases) }\end{array}$} & \multirow{2}{*}{$\begin{array}{l}\text { Group B } \\
\text { ( } n=177 \\
\text { cases) }\end{array}$} & & \multirow{2}{*}{$\begin{array}{l}\text { Group A } \\
(n=29 \\
\text { cases) }\end{array}$} & \multirow{2}{*}{$\begin{array}{l}\text { Group B } \\
\text { (n=29 } \\
\text { cases) }\end{array}$} & \\
\hline & & & & & & & \\
\hline \multirow[t]{2}{*}{ Gender } & Male & 4 & 29 & \multirow[t]{2}{*}{0.625} & 4 & 4 & \multirow{2}{*}{$\overrightarrow{0.99}$} \\
\hline & Female & 27 & 148 & & 25 & 25 & \\
\hline \multicolumn{2}{|l|}{ Age (yr) } & $67.2 \pm 5.5$ & $62.9 \pm 7.8$ & 0.004 & $66.8 \pm 5.5$ & $68.8 \pm 6.6$ & 0.218 \\
\hline \multicolumn{2}{|c|}{$\mathrm{BMI}\left(\mathrm{kg} / \mathrm{m}^{2}\right)$} & $24.8 \pm 3.3$ & $25.1 \pm 3.2$ & 0.577 & $24.8 \pm 3.4$ & $24.9 \pm 2.7$ & 0.895 \\
\hline \multicolumn{2}{|c|}{ BMD (T-score) } & $-1.8 \pm 0.7$ & $-0.8 \pm 1.3$ & 0.000 & $-1.7 \pm 0.7$ & $-1.6 \pm 0.7$ & 0.65 \\
\hline \multicolumn{2}{|l|}{$\operatorname{PI}\left({ }^{\circ}\right)$} & $45.1 \pm 12$ & $46.7 \pm 11.8$ & 0.486 & $45.9 \pm 11.8$ & $48.3 \pm 11.6$ & 0.428 \\
\hline \multicolumn{2}{|c|}{ Preoperative-SVA (mm) } & $60.7 \pm 32.2$ & $58.5 \pm 32.8$ & 0.733 & $59 \pm 31.2$ & $64.2 \pm 27.9$ & 0.511 \\
\hline \multicolumn{2}{|c|}{ Postoperative-SVA (mm) } & $43.2 \pm 31.1$ & $33.6 \pm 26.7$ & 0.072 & $39.2 \pm 27.8$ & $38 \pm 28.8$ & 0.864 \\
\hline \multicolumn{2}{|c|}{ Preoperative-LL $\left(^{\circ}\right)$} & $-24.1 \pm 7.2$ & $-28.9 \pm 9.5$ & 0.009 & $-24 \pm 7.4$ & $-24.6 \pm 8.2$ & 0.75 \\
\hline \multicolumn{2}{|c|}{ Postoperative-LL $\left({ }^{\circ}\right)$} & $-34.7 \pm 7.9$ & $-37.2 \pm 9.8$ & 0.184 & $-34.8 \pm 7.9$ & $\begin{array}{l}-36.9 \pm \\
10.7\end{array}$ & 0.388 \\
\hline \multicolumn{2}{|c|}{ Preoperative-PT $\left(^{\circ}\right)$} & $25.7 \pm 8.1$ & $28.9 \pm 9.5$ & 0.082 & $25.8 \pm 8.3$ & $24.6 \pm 8.2$ & 0.58 \\
\hline \multicolumn{2}{|c|}{ Postoperative-PT $\left({ }^{\circ}\right)$} & $18.2 \pm 5.6$ & $21.4 \pm 7.2$ & 0.021 & $18.6 \pm 5.5$ & $17.9 \pm 5.6$ & 0.638 \\
\hline \multicolumn{2}{|c|}{$\begin{array}{l}\text { Postoperative-PI minus } \\
\text { LL }\left({ }^{\circ}\right)\end{array}$} & $10.4 \pm 13.3$ & $9.5 \pm 13.2$ & 0.737 & $11.1 \pm 13.5$ & $11.4 \pm 14.1$ & 0.932 \\
\hline \multicolumn{2}{|c|}{ Preoperative $-\mathrm{TK}\left({ }^{\circ}\right)$} & $34.8 \pm 6.4$ & $36.7 \pm 11.1$ & 0.363 & $35.1 \pm 6.5$ & $35.2 \pm 10.4$ & 0.964 \\
\hline \multicolumn{2}{|c|}{ Postoperative -TK $\left(^{\circ}\right)$} & $37.2 \pm 5.1$ & $35.5 \pm 8.8$ & 0.3 & $37.4 \pm 4.9$ & $35.1 \pm 7.4$ & 0.166 \\
\hline \multicolumn{2}{|c|}{$\begin{array}{l}\text { Instrumented vertebrae } \\
\text { (no.) }\end{array}$} & $8.8 \pm 0.8$ & $8.7 \pm 0.8$ & 0.835 & $8.8 \pm 0.9$ & $8.8 \pm 0.8$ & 0.878 \\
\hline \multicolumn{2}{|c|}{$\begin{array}{l}\text { Three column } \\
\text { osteotomy (no.) }\end{array}$} & 5 & 22 & 0.572 & 5 & 3 & 0.703 \\
\hline \multicolumn{2}{|c|}{ Double rods (no.) } & 3 & 13 & 0.653 & 3 & 1 & 0.611 \\
\hline \multirow[t]{2}{*}{ UIV (no.) } & T8 or above & 1 & 15 & \multirow[t]{2}{*}{0.476} & 0 & 0 & \multirow[t]{2}{*}{--} \\
\hline & T9 or below & 30 & 162 & & 29 & 29 & \\
\hline
\end{tabular}

Below L5: S1 and (or) S2-alar-iliac screws, BMI: body mass index, BMD: bone mineral density, PI: pelvic incidence, PT: pelvic tilt, LL: lumbar lordosis, SVA: sagittal vertical axis, TK: thoracic kyphosis. 


\begin{tabular}{|c|c|c|c|c|c|c|c|}
\hline \multicolumn{2}{|l|}{ Variable } & \multicolumn{2}{|l|}{ All cases } & \multirow{3}{*}{$\underset{\text { value }}{p}$} & \multicolumn{2}{|c|}{ Matched group* } & \multirow{3}{*}{$\begin{array}{l}p \\
\text { value }\end{array}$} \\
\hline & & Group A & Group B & & Group A & Group B & \\
\hline & & $\begin{array}{l}(n=31 \\
\text { cases) }\end{array}$ & $\begin{array}{l}(n=177 \\
\text { cases) }\end{array}$ & & $\begin{array}{l}(n=29 \\
\text { cases) }\end{array}$ & $\begin{array}{l}(n=29 \\
\text { cases) }\end{array}$ & \\
\hline \multirow[t]{2}{*}{ LIV (no.) } & L5 or above & 23 & 121 & \multirow{2}{*}{$\hat{0}_{0.001}$} & 21 & 20 & \multirow[t]{2}{*}{0.773} \\
\hline & Below L5 & 8 & 56 & & 8 & 9 & \\
\hline
\end{tabular}

\section{Propensity Score-matched Two Groups}

In this study, there were 58 propensity-matched patients in group A and B with similar parameters. There were 4 males and 25 females in group $A(n=29)$ and the same sex proportion in group $B(n=29)$. Age, $\mathrm{BMI}, \mathrm{BMD}, \mathrm{PI}$, preoperative and early postoperative PT, LL, PI minus LL, SVA and TK showed no significant differences between the two groups. In addition, number of instrumented vertebrae and the frequency of UIV and LIV showed no significant difference between two groups $(p=0.878, p>0.99$ and $p=0.773$ respectively). All these data indicated that propensity score matching had eliminated the confounding factors and thus both two groups were comparable (Table 1).

After propensity score matching, the common characteristic of both groups was low BMD (T-score: $-1.7 \pm$ 0.7 vs $-1.6 \pm 0.7, p=0.65$ ). The postoperative complications of both groups were summarized in Table 2 .

The incidence of PJK showed no significantly difference between group A and B (PJK 10.3\% vs 13.8\%, $p$ > 0.99). Compared to group $A$, the group $B$ had a higher proportion of patients developing proximal junctional failure $(24.1 \%$ vs $0 \%, p=0.01)$. Pedicle screw loosening at UIV and (or) LIV showed no difference $(24.1 \%$ vs $3.4 \%, p=0.052)$ between two groups. In group $\mathrm{A}$, cement leakage was detected in five cases via AP and lateral fluoroscopy during surgery. These five cases with peri-vertebral cement leakage had no neurological deficit or pulmonary cement embolism that was confirmed by postoperative $X$-ray. One case in group A underwent revision surgery due to the onset of neurological deficit which was caused by thoracic intervertebral disc failure and stenosis between UIV and UIV +1 . Seven cases in group $B$ had developed into PJF while six cases of them underwent revision surgery (4 cases with fracture of UIV $+1,2$ case with fracture of UIV and UIV +1) (Fig. 3). 
Table 2

The postoperative complications of both groups

\begin{tabular}{|c|c|c|c|c|c|c|}
\hline \multirow[t]{3}{*}{ Variable } & \multicolumn{2}{|l|}{ All cases } & \multirow{3}{*}{$\begin{array}{l}p \\
\text { value }\end{array}$} & \multicolumn{2}{|c|}{ Matched group* } & \multirow{3}{*}{$\begin{array}{l}p \\
\text { value }\end{array}$} \\
\hline & Group A & Group B & & Group A & Group B & \\
\hline & $\begin{array}{l}(\mathrm{n}=31 \\
\text { cases })\end{array}$ & $\begin{array}{l}(n=177 \\
\text { cases) }\end{array}$ & & $\begin{array}{l}(n=29 \\
\text { cases) }\end{array}$ & $\begin{array}{l}(n=29 \\
\text { cases) }\end{array}$ & \\
\hline PJK (no. [\%]) & $4(12.9)$ & $27(15.3)$ & 0.948 & $3(10.3)$ & $4(13.8)$ & $\overrightarrow{0.99}$ \\
\hline PJF (no. [\%]) & 0 & $24(13.6)$ & 0.029 & 0 & $7(24.1)$ & 0.01 \\
\hline $\begin{array}{l}\text { Screw loosening at UIV } \\
\text { and (or) LIV }\end{array}$ & $1(3.2)$ & $21(11.9)$ & 0.211 & $1(3.4)$ & $7(24.1)$ & 0.052 \\
\hline Cement leakage (no. [\%]) & $5(16.1)$ & -- & -- & $5(17.2)$ & 0 & -- \\
\hline $\begin{array}{l}\text { Neurological complication } \\
\text { (no. [\%]) }\end{array}$ & 0 & 1 & -- & 0 & 0 & -- \\
\hline Pulmonary embolism & 0 & 1 & -- & 0 & 0 & -- \\
\hline
\end{tabular}

\section{Discussion}

In surgical correction of ASD, high load bearing requirements for the pedicular screw/bone interface is essential for rigid posterior internal fixation system, which is important for spinal fusion. Bone quality is crucial to pedicle screw performance ${ }^{17}$. However, elderly patients with ASD are commonly accompanied with low BMD or osteoprosis in clinical practice. It has been proven that osteoporotic bone can increase the risk of implants failure ${ }^{17}$. Patients with low BMD or osteoporosis are more likely to suffer from PJK/PJF ${ }^{6,18,19}$. A propensity score matching study showed that low BMD was a significant risk factor for PJF with a odds ratio of $6.4^{6}$, which was consistent with our present study. After propensity score matching, the result showed that patients in group B with low BMD or osteoporosis were more inclined to develop into PJF or pedicle screws loosening compared to the patients in group A with "normal bone quality" (whose vertebrae was reinforced with PMMA cement).

In the last two decades, pedicle screw cement augmentation has been used to increase fatigue strength and kyphoplasty or verterbraplasty is commonly used to treat osteoporotic vertebrae fracture ${ }^{12,20}$. In management of ASD with low BMD or osteoporosis, prophylactic verterbraplasty (PV) at UIV +1 and (or) UIV was proposed to prevent PJK/PJF. Robert A Hart, et al ${ }^{20}$ firstly reported that 15 patients with osteoporosis (underwent PV at UIV + 1) did not suffer from proximal junctional acute collapse after long posterior lumbar fusion and $\mathrm{PV}$ was considered as a cost-effective intervention. Later, Aydogan, $\mathrm{M}$, et al ${ }^{11}$ reported that 36 patients, who underwent pedicle screw fixation with vertebroplasty and PV at UIV +1 and LIV, with bone T-score lower than -2.5 had no proximal and distal junctional fractures. Furthermore, the reports of Ghobrial GM, et al ${ }^{7}$ and Martin CT, et al ${ }^{10}$ supported that prophylactic vertebroplasty (PMMA at 
the UIV and UIV + 1) could prevent PJK and PJF. However, the efficacy of prophylactic cement augmentation in the treatment of ASD was not supported by recent studies ${ }^{8,13}$. These results showed that bone cement reinforcement at UIV and UIV + 1 cannot prevent PJK/PJF. In our current study, the result of both pre- and post-propensity scoring matching showed that combination of CAFPS and PV at UIV + 1 could prevent PJF, but could not reduce the incidence of PJK. It suggested that cement augmenttation could not reduce the risk of disc failure, disruption of the posterior osseo-ligamentous complex and fragile paraspinal muscle, which might cause PJK.

The reasons for the controversy about whether prophylactic cement augmentation could minimize the risk for PJK and PJF were as follows. Firstly, multiple risk factors could be correlated with PJK and PJF, previous studies did not eliminate the heterogeneity of ASD population between groups, such as age, BMD or spino-pelvic parameters. In this study, we carried out the propensity score matching analysis aiming to minimize the heterogeneity of multiple risk factors causing the PJK and PJF and also to avoid selection bias. Our results showed that both two groups were comparable after propensity score matching (Table 2). Secondly, there was still no consensus on the definition of PJK or PJF, which might be another factor that influence the evaluation of prophylactic cement augmentation. The previous definition of PJF is a progressive form of the PJK or the symptomatic PJK that requires surgery ${ }^{1,2}$. However, according to our experience, some cases with acute proximal junctional frature did no present the progressive form of the PJK. Therefore, in our study we adjusted definition of PJK and PJF based on previous articles $^{1-3}$.

In surgical treatment of patients with osteoporosis, cement-augmented fenestrated pedicle screw (CAFPS) had been safely used ${ }^{15,21}$. CAFPS could provide comparable screw stability to solid pedicle screws augmented with high-viscosity cement ${ }^{22}$. In our study, the CAFPS did not develop into screw pullout from the vertebrae, but one case in group A had pedicle screw loosening at LIV. Our result indicated that CAFPS might not prevent the pedicle screw loosening of ASD patients with osteoporosis treated by long posterior instrumented fusion.

The complication of cement leakage had received high concern. The high risks for cement leakage after vertebroplasty or kyphoplasty included intravertebral cleft, cortical disruption, low cement viscosity and high volume of injected cement ${ }^{23}$. Previous report indicated that the risk for CAFPS relevant symptomatic pulmonary cement embolism included CAFPS used in more than 7 vertebrae or more than 14 fenestrated screws, severe osteoporosis and the total cement volume more than 20 to $25 \mathrm{ml}$. In this study, no cases used more than $20 \mathrm{ml}$ cement volume in total and PMMA cement volume for per vertebrae was less than the critical value reported previously ${ }^{15,23}$. Besides, the vertebroplasty was prophylactic treatment without the risk of intravertebral cleft or cortical disruption. Therefore, no cases in group A have complications correlated with cement leakage, though peri-vertebral cement leakage in 5 cases was detected. Thus, it could be safe to use CAFPS and PV in the management of ASD with osteoporosis.

There are some limitations in this study. Firstly, the potential weakness of this study was its relatively small sample size $(n=58)$. Future studies with a larger sample size could yield more accurate results. 
Secondly, though this propensity score matched cohort analysis minimized the heterogeneity of ASD, some potential risk factors for PJK or PJF were not commonly assessed, such as prevalence of sarcopenia in middle-aged and elderly women ${ }^{24}$ and disruption of the posterior osseo-ligamentous complex.

\section{Conclusion}

Surgeons have paid high attention to the prevention of postoperative PJK and PJF in patients with long posterior spinal fusions. This propensity score-matched study indicated that combined application of CAFPS and PV could not prevent the development of PJK, but could reduce the incidence of PJF in the surgical management of ASD with low BMD or osteoporosis.

\section{Abbreviations}

PV

prophylactic vertebroplasty

CAFPS

cement-augmented fenestrated pedicle screw

ASD

adult spinal deformity

PJK

Proximal junctional kyphosis

PJF

proximal junctional failure

UIV

uppermost instrumented vertebrae

LIV

lowest instrumented vertebrae

BMI

body mass index

BMD

bone mineral density

PSM

Propensity score matching

SVA

sagittal vertical axis

PMMA

polymethylmethacrylate

$\mathrm{PI}$

pelvic incidence 
PT

pelvic tilt

LL

lumbar lordosis

TK

thoracic kyphosis.

\section{Declarations}

\section{Ethics approval and consent to participate:}

our research is a retrospective study, which used medical records obtained from patients' imaging data and clinical data. Thus, the ethical approval is exempted from the Ethical Committee of our institutions.

Consent for publication:

Consent for publication was obtained from all participants.

\section{Availability of data and materials:}

the datasets used and/or analysed during the current study are available from the corresponding author on reasonable request.

Competing interests:

the authors declare that they have no competing interests" in this section.

\section{Funding:}

The authors report no conflict of interest concerning the materials or methods used in this study or the findings specified in this paper.

\section{Authors' contributions:}

Xuesong Zhang has made contribution to the conception and design of the work; Qi Wang, Wenhao Hu and Fanqi Hu have made contribution to the data acquisition and analysis, Weibo Liu, Teng Li and Shiqi Cao have made contribution to interpretation of data; Qi Wang and Chi Wang were major contributors in writing the manuscript. All authors read and approved the final manuscript

\section{Acknowledgements:}


Not applicable.

\section{References}

1. Lee J, Park YS. Proximal Junctional Kyphosis: Diagnosis, Pathogenesis, and Treatment. Asian Spine J. 2016;10:593-600.

2. Hyun SJ, Lee BH, Park JH, et al. Proximal Junctional Kyphosis and Proximal Junctional Failure Following Adult Spinal Deformity Surgery. Korean J Spine. 2017;14:126-32.

3. Yilgor C, Sogunmez N, Boissiere L, et al. Global Alignment and Proportion (GAP) Score: Development and Validation of a New Method of Analyzing Spinopelvic Alignment to Predict Mechanical Complications After Adult Spinal Deformity Surgery. J Bone Joint Surg Am. 2017;99:1661-72.

4. Safaee MM, Deviren V, Dalle Ore C, et al. Ligament augmentation for prevention of proximal junctional kyphosis and proximal junctional failure in adult spinal deformity. J Neurosurg Spine. 2018;28:512-9.

5. Line BG, Bess S, Lafage R, et al. Effective Prevention of Proximal Junctional Failure in Adult Spinal Deformity Surgery Requires a Combination of Surgical Implant Prophylaxis and Avoidance of Sagittal Alignment Overcorrection. Spine (Phila Pa 1976). 2020;45:258-67.

6. Yagi M, Fujita N, Tsuji O, et al. Low Bone-Mineral Density Is a Significant Risk for Proximal Junctional Failure After Surgical Correction of Adult Spinal Deformity: A Propensity Score-Matched Analysis. Spine (Phila Pa 1976. 2018;43:485-91.

7. Ghobrial GM, Eichberg DG, Kolcun JPG, et al. Prophylactic vertebral cement augmentation at the uppermost instrumented vertebra and rostral adjacent vertebra for the prevention of proximal junctional kyphosis and failure following long-segment fusion for adult spinal deformity. Spine $\mathrm{J}$. 2017;17:1499-505.

8. Raman T, Miller E, Martin CT, et al. The effect of prophylactic vertebroplasty on the incidence of proximal junctional kyphosis and proximal junctional failure following posterior spinal fusion in adult spinal deformity: a 5-year follow-up study. Spine J. 2017;17:1489-98.

9. Theologis AA, Burch S. Prevention of Acute Proximal Junctional Fractures After Long Thoracolumbar Posterior Fusions for Adult Spinal Deformity Using 2-level Cement Augmentation at the Upper Instrumented Vertebra and the Vertebra 1 Level Proximal to the Upper Instrumented Vertebra. Spine (Phila Pa 1976). 2015;40:1516-26.

10. Martin CT, Skolasky RL, Mohamed AS, et al. Preliminary Results of the Effect of Prophylactic Vertebroplasty on the Incidence of Proximal Junctional Complications After Posterior Spinal Fusion to the Low Thoracic Spine. Spine Deform. 2013;1:132-8.

11. Aydogan M, Ozturk C, Karatoprak O, et al. The pedicle screw fixation with vertebroplasty augmentation in the surgical treatment of the severe osteoporotic spines. J Spinal Disord Tech. 2009;22:444-7. 
12. Singh V, Mahajan R, Das K, et al. Surgical Trend Analysis for Use of Cement Augmented Pedicle Screws in Osteoporosis of Spine: A Systematic Review (2000-2017). Global Spine J. 2019;9:78395.

13. Han S, Hyun SJ, Kim KJ, et al. Effect of Vertebroplasty at the Upper Instrumented Vertebra and Upper Instrumented Vertebra +1 for Prevention of Proximal Junctional Failure in Adult Spinal Deformity Surgery: A Comparative Matched-Cohort Study. World Neurosurg 2019.

14. Kane LT, Fang T, Galetta MS, et al. Propensity Score Matching: A Statistical Method. Clin Spine Surg. 2020;33:120-2.

15. Ulusoy OL, Kahraman S, Karalok I, et al. Pulmonary cement embolism following cement-augmented fenestrated pedicle screw fixation in adult spinal deformity patients with severe osteoporosis (analysis of 2978 fenestrated screws). Eur Spine J. 2018;27:2348-56.

16. Zou D, Muheremu A, Sun Z, et al. Computed tomography Hounsfield unit-based prediction of pedicle screw loosening after surgery for degenerative lumbar spine disease. J Neurosurg Spine 2020:1-6.

17. Hoppe S, Keel MJ. Pedicle screw augmentation in osteoporotic spine: indications, limitations and technical aspects. Eur J Trauma Emerg Surg. 2017;43:3-8.

18. Kim JS, Phan K, Cheung ZB, et al. Surgical, Radiographic, and Patient-Related Risk Factors for Proximal Junctional Kyphosis: A Meta-Analysis. Global Spine J. 2019;9:32-40.

19. Zou L, Liu J, Lu H. Characteristics and risk factors for proximal junctional kyphosis in adult spinal deformity after correction surgery: a systematic review and meta-analysis. Neurosurg Rev. 2019;42:671-82.

20. Hart RA, Prendergast MA, Roberts WG, et al. Proximal junctional acute collapse cranial to multi-level lumbar fusion: a cost analysis of prophylactic vertebral augmentation. Spine J. 2008;8:875-81.

21. Wang W, Liu C, Li J, et al. Comparison of the fenestrated pedicle screw and conventional pedicle screw in minimally percutaneous fixation for the treatment of spondylolisthesis with osteoporotic spine. Clin Neurol Neurosurg. 2019;183:105377.

22. Leichtle $\mathrm{Cl}$, Lorenz $\mathrm{A}$, Rothstock $\mathrm{S}$, et al. Pull-out strength of cemented solid versus fenestrated pedicle screws in osteoporotic vertebrae. Bone Joint Res. 2016;5:419-26.

23. Zhan Y, Jiang J, Liao H, et al. Risk Factors for Cement Leakage After Vertebroplasty or Kyphoplasty: A Meta-Analysis of Published Evidence. World Neurosurg. 2017;101:633-42.

24. Moskven E, Bourassa-Moreau E, Charest-Morin R, et al. The impact of frailty and sarcopenia on postoperative outcomes in adult spine surgery. A systematic review of the literature. Spine $\mathrm{J}$. 2018;18:2354-69.

\section{Figures}




\section{Inclusion criteria :}

- Age $\geq 20$ years

- lumbar scoliosis $>20^{\circ}$, pelvic tilt $(\mathrm{PT})>25^{\circ}$ or sagittal vertical axis (SVA) $>5 \mathrm{~cm}$

- Instrumented spinal fusion $>5$ vertebral levels and uppermost instrumented vertebrae at T11 or above

- posterior spinal fusion with bilateral pedicle-screw fixation and at least 2 years follow-up

Exclusion criteria:

- hook and/or sublaminar wiring usage

- long-term use of glucocorticoids or renal dysfunction

- neurological disorders affecting balance

- the use of anti-osteoporotic drugs pre- or post-operatively

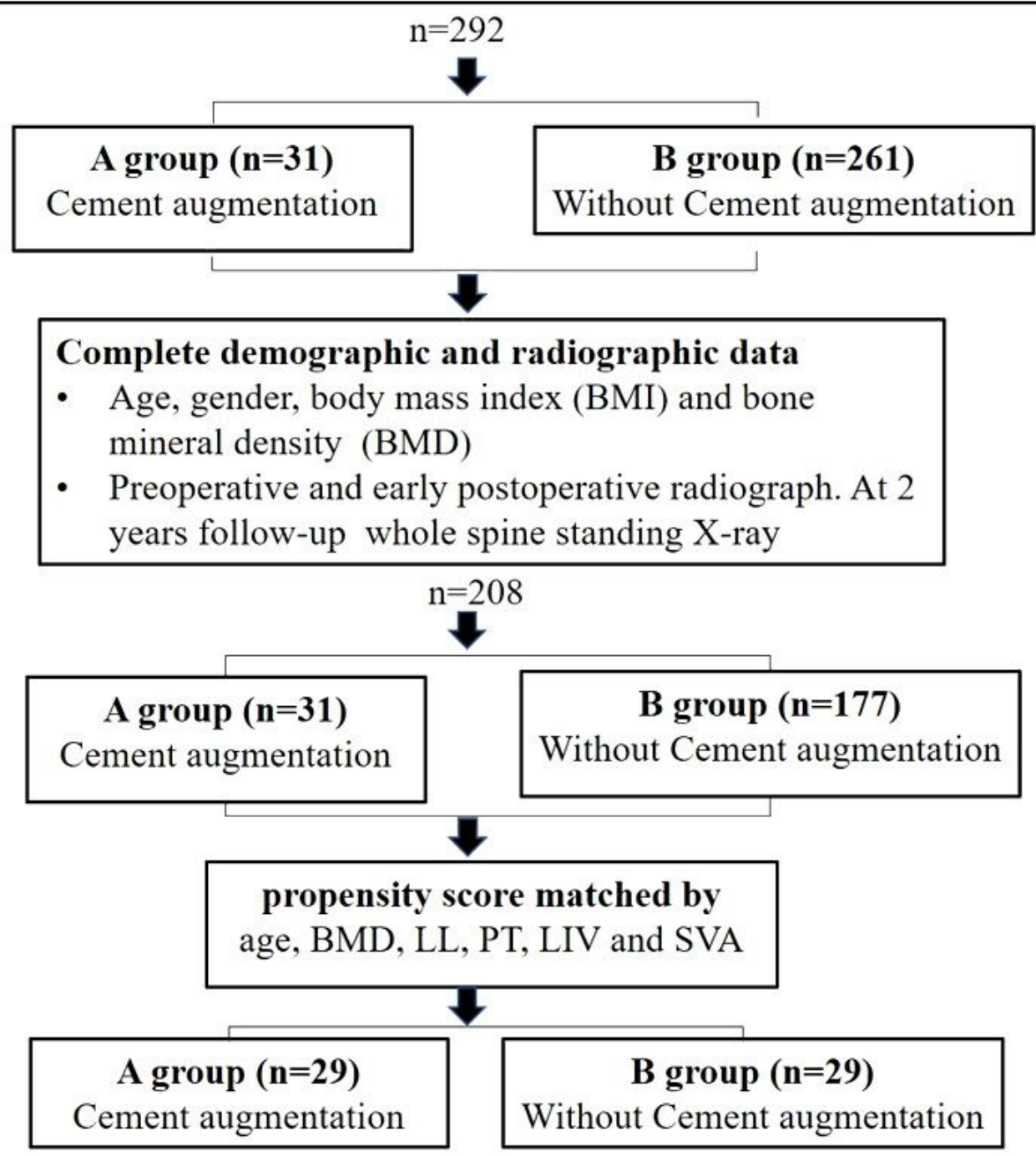

Figure 1

Flow chart of the process for propensity score-matched design. 

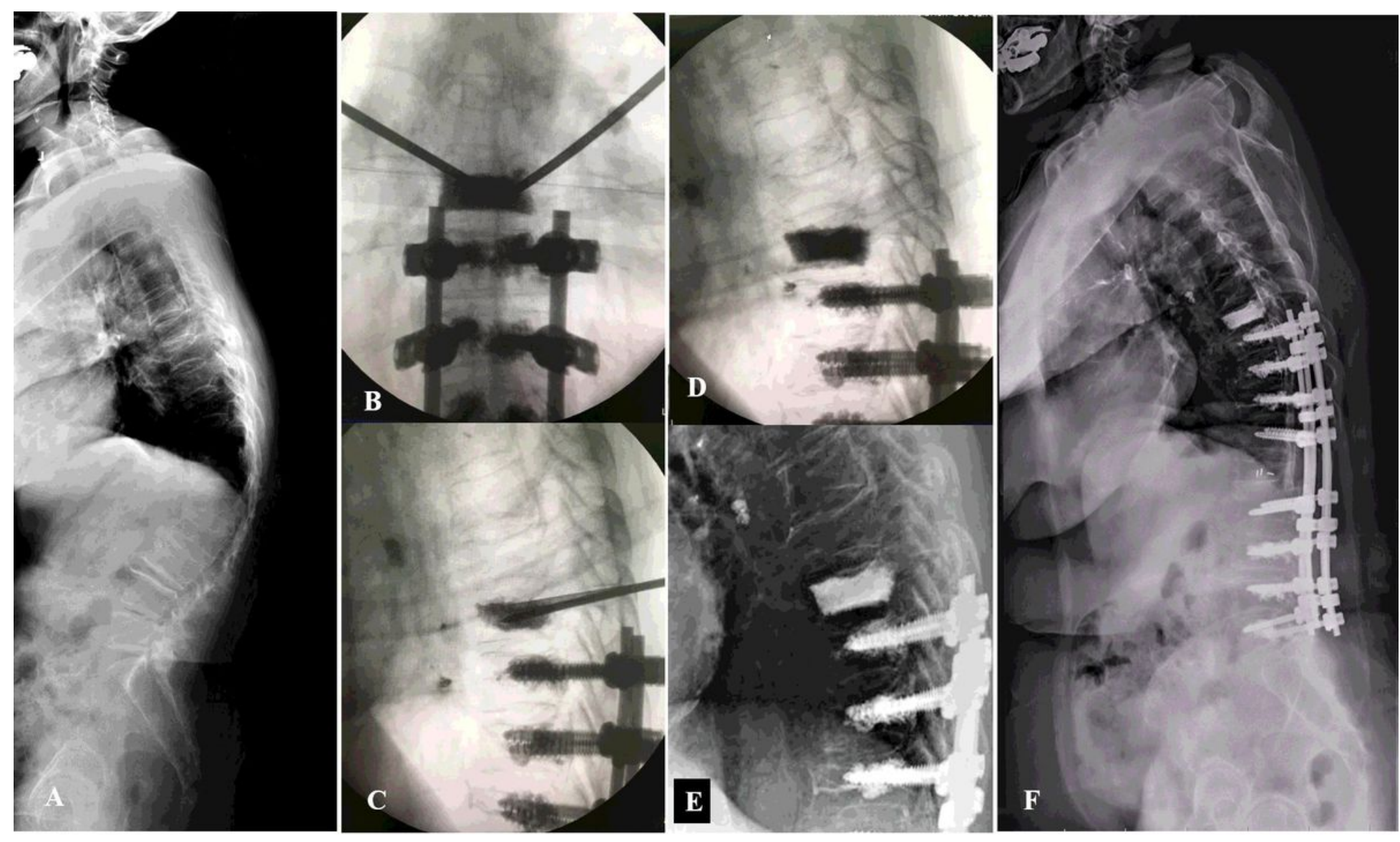

Figure 2

A 71 years old woman underwent posterior spinal fusion from T9 to L5 with cement-augmented fenestrated pedicle screw (CAFPS) and prophylactic vertebroplasty (PV) at UIV+1. A. Preoperative wholespine lateral radiograph. B, C, D. PV at UIV+1 was visualized with fluoroscopic guidance and there is no image finding of cement leakage. E. Early postoperative lateral radiograph showed proximal junctional angle was $7^{\circ}$. F. The final follow-up lateral radiograph showed proximal junctional angle was $18^{\circ}$, proximal junctional kyphosis was detected. This patient was asymptomatic. 

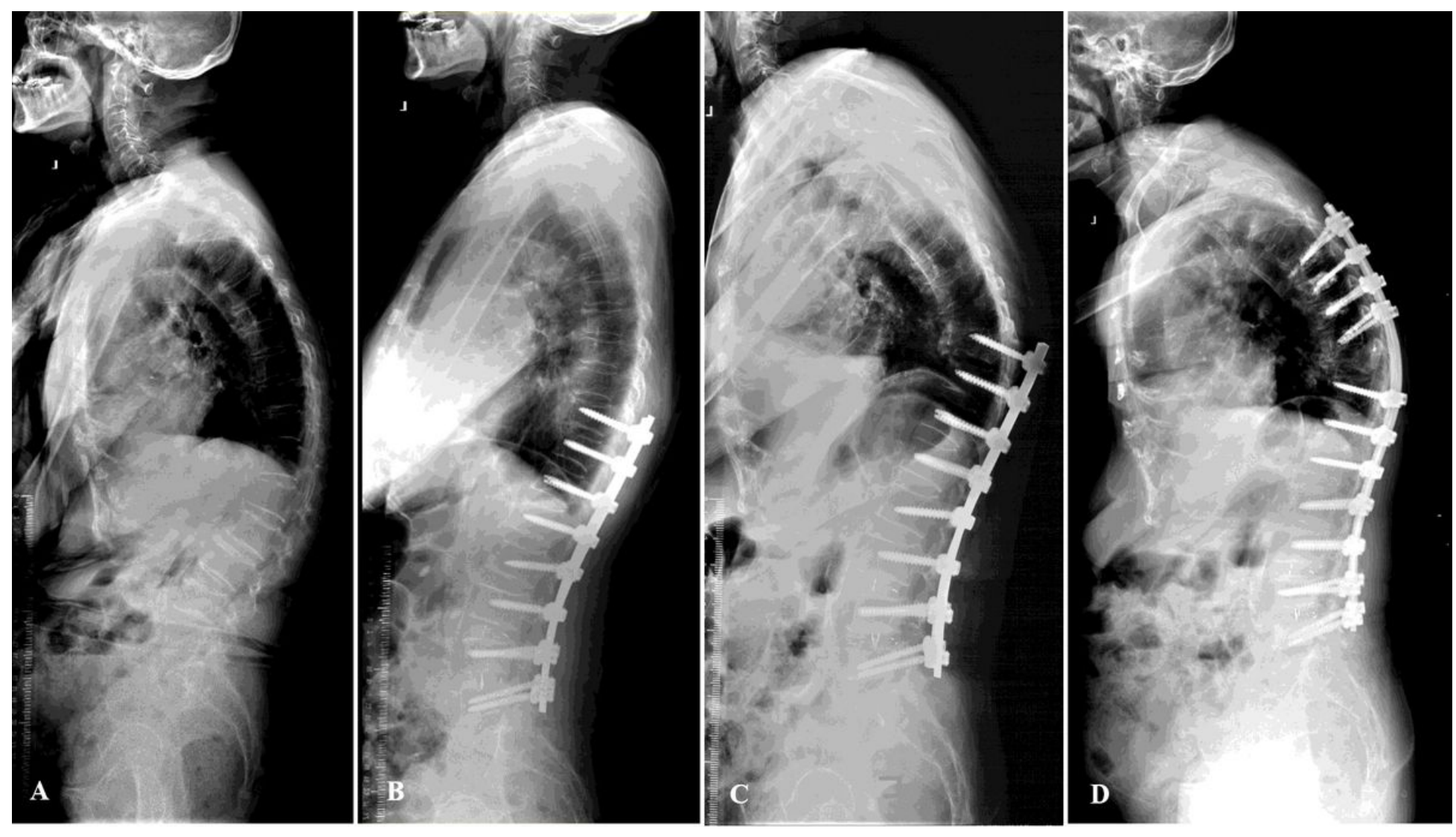

\section{Figure 3}

A, B. A 73 years old woman with adult spinal deformity underwent posterior spinal fusion from T10 to L5. C. Vertebral fracture at UIV+1, dislodgment of pedicle screws at UIV and sagittal subluxation occurred 9 months later after spinal deformity surgery. D. The revision surgery was carried out to manage the junctional problem. 